\title{
A Technique for Laparoendoscopic Resection of Posterior Fundic Gastric GISTs Without Need for a Gastrotomy
}

\author{
Ajay V. Maker, MD, FACS \\ Division of Surgical Oncology, Department of Surgery, University of Illinois at Chicago, Chicago, Il
}

\begin{abstract}
Background. The majority of gastrointestinal stromal tumors (GISTs) are located in the stomach. With greater experience in minimally invasive oncologic surgery, gastric GISTs are being increasingly approached laparoscopically. Posterior proximally located endophytic gastric GISTs can be challenging to approach laparoscopically and excise with an adequate margin without an anterior or posterior gastrotomy, or intragastric ports.

Methods. The gastrocolic and gastrosplenic ligaments are divided up to the gastroesophageal junction. The left lateral segment of the liver is mobilized to allow anterior reflection of the gastric fundus. Intraoperative ultrasound confirms the location and extent of the tumor base. Upper endoscopy is performed to trans-illuminate, confirm tumor location, and search for multifocality. Traction sutures are placed around the tumor to distract endophytic lesions. With organo-axial rotation of the stomach using the stay
\end{abstract}

sutures, an endoGIA stapler approximates the posterior fundic wall under the base of the lesion ensuring an adequate margin and eliminating the risk of gastric spillage. Appropriate stapler placement and margins are assisted real-time endoscopically with picture-in-picture. The stapleline is tested for leaks and inspected for hemostasis laparoendoscopically.

Results. Complete resection of GISTs with adequate margins is performed with sound oncologic principles and demonstrated in tumors of varying sizes and locations in the proximal posterior stomach, including near the GE junction.

Conclusions. This video demonstrates a simple laparoendoscopic technique to quickly localize even small tumors, visually confirm adequate margins, and excise gastric GISTs without spillage or gastrotomy that are located in a typically difficult area of the stomach to approach laparoscopically.

This video was presented in part at the "top-videos" session of the 2013 SSO annual meeting in Washington D.C.

Electronic supplementary material The online version of this article (doi:10.1245/s10434-013-3199-8) contains supplementary material, which is available to authorized users.

(C) Society of Surgical Oncology 2013

First Received: 17 March 2013;

Published Online: 14 August 2013

A. V. Maker, MD, FACS

e-mail: amaker@uic.edu 